\title{
A General Approach for Solving Assignment Problems Involving with Fuzzy Cost Coefficients
}

\author{
P. K. De \\ Department of Mathematics, National Institute of Technology, India \\ E-mail: pijusde@rediffmail.com
}

Bharti Yadav

Department of Mathematics, Krishna Institute of Engineering and Technology, India

E-mail: bharti1406@rediffmail.com

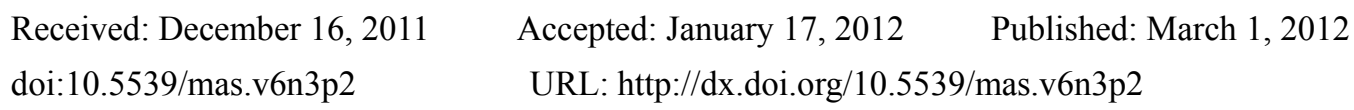

\begin{abstract}
Assignment problem is one of the most-studied, well known and important problems in mathematical programming. In this paper two different type of assignment problems are discussed: conventional and fuzzy assignment problem. In conventional assignment problem, cost is always certain. This paper develops an approach to solve the fuzzy assignment problem where cost is not deterministic numbers but imprecise ones. Here, the elements of the cost matrix of the assignment problem are triangular fuzzy numbers. Its triangular shaped membership function is defined. The optimal solution of fuzzy assignment problem is obtained successfully by using this approach. Compared with the result of conventional assignment problem, the result obtained by our approach is more advantaged for decision-makers. Finally, to show the efficiency of the proposed approach, the problem is demonstrated by one numerical example.
\end{abstract}

Keywords: Fuzzy sets, Fuzzy mathematical programming, Assignment problem, Triangular fuzzy number

\section{Introduction}

The assignment problem (AP) is a special type of linear programming problem in which our objective is to assign a number of jobs to an equal number of persons, so as to minimize the total assignment cost or to minimize the total consumed time for execution of all the jobs. However, much of decision-making in the real world takes place in an environment where the objectives, constraints or parameters are not precise. Therefore, a decision is often made on the basis of vague information or uncertain data. In 1970, Bellmann and Zadeh introduced the concepts of fuzzy set theory into the decision-making problems involving uncertainty and imprecision. Fuzzy assignment problems have received great attention in recent years. Lin and Wen (2004) proposed a labeling algorithm for solving fuzzy assignment problems. Yaakob and Watada (2009) proposed the fuzzy approach for solving assignment problem, in which they presented a worker's placement model capable of evaluating worker's suitability for a specified task according their performance, social and mental factor. Yang and Liu (2005) designed a tabu search algorithm based on fuzzy simulation to achieve an appropriate best solution of fuzzy assignment problem. Chen (1985) proved some theorems and proposed a fuzzy assignment model which did not consider the differences of individuals. Wang (1987) solved a similar model by graph theory. Sakawa (2001) dealt with actual problems on production and work force assignment in a housing material manufacturer and a subcontract firm and formulated two kinds of two level programming problems. Applying the interactive fuzzy programming for two-level linear and linear fractional programming problems, they desired satisfactory solutions to the problems and therefore compared the results.

Liu and Gao (2009) proposed an equilibrium optimization problem and extended the assignment problem to the equilibrium multi-job assignment problem, equilibrium multi-job quadratic assignment problem and used genetic algorithm to solve the proposed models. Majumdar and Bhunia (2007) proposed an elitist genetic algorithm to solve the generalized assignment problem with imprecise cost/time. Ye and $\mathrm{Xu}$ (2008) proposed an effective method on priority-based genetic algorithm to solve fuzzy vehicle routing assignment when there is no genetic algorithm which can give clear procedure of solving it. The linear interactive and discrete optimization [LINDO] 
(1984) general interactive optimizer [GINO] (1986) and TORA packages (1992) as well as many other commercial and academic packages are useful in finding the solution of the assignment problems.

In this paper, we are proposing a new approach to find the optimal solution of fuzzy assignment problems by representing cost parameters as triangular fuzzy numbers. To illustrate the proposed approach a fuzzy assignment problem is solved and the obtained results are discussed.

This paper is organized as follows: In section 2, some basic definitions and arithmetic operations are reviewed. In section 3, formulation of fuzzy assignment problem is described. In section 4, a new approach is proposed to find the optimal solution of fuzzy assignment problem. Section 5, presents a numerical example to illustrate the proposed approach. The results are discussed in section 6 and section 7 gives few concluding remarks on the proposed approach.

\section{Fuzzy Preliminaries}

The terms of expression such as "very good", "really good", "not bad" and "rather clear" are used very often in daily life, common that they are more or less tainted with fuzziness. With different daily decision-making problems of diverse intensity, the results can be misleading if the fuzziness of human decision-making is not taken into account. The theory of fuzzy set is based upon the investigation reported by Bellmann and Zadeh (1970), involves a mathematical description of vague (inexact, fuzzy) elements, with the vagueness of information resulting not from the stochastic character of the system, but from the lack of uniqueness or selectivity that of. Accordingly, the answer to the question whether an element is associated with a fuzzy set will not be in the form of a YES-OR-NO decision but it will require carefully graded judgment of its association. The degree of association of defined elements is determined by an association function that must come within the scope of particular mathematical definitions, axioms and operational rules.

Fuzzy set is a theory of graded concept, has a vague boundary set, as compared to with crisp set. This is also a powerful modeling language that can cope with a large fraction of uncertainties of real life situations.

In this section, some basic definitions and arithmetic operations are reviewed.

\subsection{Basic Definitions}

Definition 1. A fuzzy set is a set whose boundary is not clear, whose elements are characterized by a membership function. Let $X$ be a universal set. A fuzzy set $\widetilde{A}$ define on $X$. A set of order pair of element whose first element $x \in X$, second element $\mu_{\tilde{A}}(\mathrm{x})$ is the membership value of element $x$ in the set $\widetilde{A}$. It is denoted by $\widetilde{A}$ or $A$, and it defined by

$$
\begin{gathered}
\tilde{A}=\left\{\left(x, \mu_{A}(x)\right) \mid x \in X\right\} \\
\text { Where } \mu_{A}(x) \rightarrow K \\
\text { and } K \in[0,1]
\end{gathered}
$$

Definition 2. A fuzzy set $\widetilde{A}$, defined on universal set of real numbers $X$, is said to be a fuzzy number if

(i) $\widetilde{A}$ is convex set i.e., $\mu_{\widetilde{A}}\left(\lambda x_{1}+(1-\lambda) x_{2}\right) \geq \min \left(\mu_{\widetilde{A}}\left(x_{1}\right), \mu_{\widetilde{A}}\left(x_{2}\right)\right), x_{1}, x_{2} \in X, \lambda \in[0,1]$;

(ii) $\tilde{A}$ is normalized fuzzy set if there exists at least one $x_{0} \in X$ with $\mu_{\widetilde{A}}\left(x_{0}\right)=1$;

(iii) it's membership function $\mu_{\widetilde{A}}(x)$ is piecewise continuous.

Definition 3. A fuzzy number $\tilde{A}=\left\{\left(x, \mu_{\widetilde{A}}(x)\right) \mid x \in X\right\}$ is non-negative if and only if $\mu_{\widetilde{A}}(x)=0$ for all $x<0$.

Definition 4. A fuzzy number $\widetilde{A}=(a, b, c)$ is said to be a triangular fuzzy number, if its membership function is given by

$$
\mu_{\tilde{\hat{A}}}(\mathrm{x})=\left\{\begin{array}{c}
0, \quad \mathrm{x}<\mathrm{a} \\
\mu_{\mathrm{A}} 1(\mathrm{x})=\frac{\mathrm{x}-\mathrm{a}}{\mathrm{b}-\mathrm{a}}, \quad \mathrm{a} \leq \mathrm{x} \leq \mathrm{b} \\
\mu_{\hat{\mathrm{A}}} \mathrm{r}(\mathrm{x})=\frac{\mathrm{c}-\mathrm{x}}{\mathrm{c}-\mathrm{b}}, \quad \mathrm{b} \leq \mathrm{x} \leq \mathrm{c} \\
0, \quad \mathrm{x}>\mathrm{c}
\end{array}\right.
$$

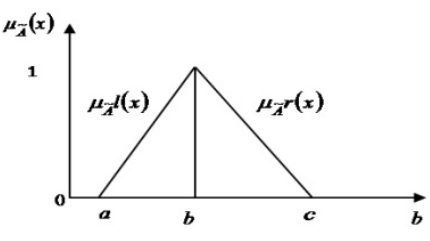

Where $\mu_{\widetilde{A}} l(x)$ and $\mu_{\widetilde{A}} r(x)=$ left membership function and right membership function of the fuzzy set $\widetilde{A}$.

Definition 5. A triangular fuzzy number $\tilde{A}=(a, b, c)$ is said to be non-negative if and only if $a \geq 0$.

Definition 6. A triangular fuzzy number $\tilde{A}=(a, b, c)$ is said to be zero triangular fuzzy number if and only if $a=0, b=0, c=0$. 
Definition 7. Two triangular fuzzy numbers $\widetilde{A}=\left(a_{1}, a_{2}, a_{3}\right)$ and $\widetilde{B}=\left(b_{1}, b_{2}, b_{3}\right)$ are said to be equal if and only if $a_{1}=b_{1}, a_{2}=b_{2}, a_{3}=b_{3}$.

\subsection{Arithmetic Operations}

Let $\widetilde{A}=\left(a_{1}, a_{2}, a_{3}\right)$ and $\widetilde{B}=\left(b_{1}, b_{2}, b_{3}\right)$ be two triangular fuzzy numbers, then arithmetic on them is defined as:

$$
\begin{gathered}
\text { Addition: } \widetilde{A}(+) \widetilde{B}=\left(a_{1}+\mathrm{b}_{1}, a_{2}+b_{2}, a_{3}+b_{3}\right) \\
\text { Subtraction: } \widetilde{A}(-) \widetilde{B}=\left(a_{1}-b_{3}, a_{2}-b_{2}, a_{3}-b_{1}\right) \\
\text { Scalar multiplication: } \lambda \widetilde{A}=\left(\lambda a_{1}, \lambda a_{2}, \lambda a_{3}\right), \text { if } \lambda \geq 0 \\
\text { Symmetric image: }-(\widetilde{A})=\left(-a_{3},-a_{2},-a_{1}\right)
\end{gathered}
$$

\section{Fuzzy Assignment Problem}

We know for every physical structure there is some mathematical phenomena and for every mathematical phenomenon there may be or may not be some physical structure. In this section we will be describing mathematical model of assignment problems in the fuzzy environment.

Assume that there are $n$ jobs and $n$ persons. $n$ Jobs must be performed by $n$ persons, where the costs depend on the specific assignments. Each job must be assigned to one and only one person and each person has to perform one and only one job. Let $C_{i j}$ be the cost if the $i$ th person is assigned the $j$ th job, the problem is to find an assignment (which job should be assigned to which person) so that the total cost for performing all jobs is minimum.

Here make a assumption that $j$ th job will be completed by $i$ th person, and let

$$
x_{i j}=\left\{\begin{array}{l}
1 \text { if } i \text { th person is assigned } j \text { th job } \\
0 \text { if } i \text { th person is not assigned } j \text { th job }
\end{array}\right.
$$

Where $x_{i j}$ denotes that $j$ th job is to be assigned to the $i$ th person.

Then, the mathematical model of assignment problem in crisp environment is:

$$
\begin{gathered}
\text { S1: } \operatorname{Min} Z=\sum_{i=1}^{n} \sum_{j=1}^{n} c_{i j} x_{i j} \\
\text { Subject to } \\
\sum_{i=1}^{\mathrm{n}} x_{i j}=1, j=1,2, \ldots, n \\
\sum_{j=1}^{\mathrm{n}} x_{i j}=1, i=1,2, \ldots, n \\
x_{i j} \in\{0,1\} \text { for } i, j=1,2, \ldots, n
\end{gathered}
$$

In above conventional assignment problem, the variables, assignment cost coefficients, are usually precise values. However, in real life situations, the parameters of assignment problem are imprecise numbers because time/cost for doing a job by a person/machine might vary due to different reasons, such as assigning men to offices, trucks to delivery routes etc. Especially the assignment cost, that which is considered as a certain value is not suitable, will be influenced directly by the above reasons. Therefore assignment cost coefficients are usually uncertain values and will change respectively in a frame. This paper considers assignment cost as a fuzzy number denoted by $\widetilde{c}=(\underline{c} / c / \bar{c})$, in that $c$ represents the most possible assignment cost, $\underline{c}$ the most optimistic assignment cost and $\bar{c}$ the most pessimistic assignment cost. If cost coefficients are fuzzy numbers, then the total assignment cost becomes fuzzy as well. Now the problem is how to achieve a minimum total cost under fuzzy cost $\widetilde{c}_{i j}$. Then, the conventional assignment problem in (1) turns into following fuzzy assignment problem.

$$
\mathrm{S} 2: \operatorname{Min} \widetilde{Z}=\sum_{i=1}^{n} \sum_{j=1}^{n} \widetilde{c}_{i j} x_{i j}
$$


Subject to

$$
\begin{aligned}
& \sum_{i=1}^{\mathrm{n}} x_{i j}=1, j=1,2, \ldots, n \\
& \sum_{j=1}^{\mathrm{n}} x_{i j}=1, i=1,2, \ldots, n \\
& x_{i j} \in\{0,1\} \text { for } i, j=1,2, \ldots, n
\end{aligned}
$$

\section{Proposed Approach}

In this section, a new approach is proposed to find the optimal solution of fuzzy assignment problems, occurring in real life situations, by representing cost coefficients as triangular fuzzy numbers. The steps of proposed approach are as follows:

\subsection{Mathematical Structure}

The fuzzy assignment problem can be stated in the form of $n \times n$ cost matrix $\tilde{c}_{i j}$ of real numbers as follows:

\begin{tabular}{llllll} 
& 1 & 2 & 3 & $j$ & $N$ \\
\hline 1 & $\widetilde{c}_{11}$ & $\widetilde{c}_{12}$ & $\widetilde{c}_{13}$ & $\tilde{c}_{1 j}$ & $\widetilde{c}_{1 n}$ \\
2 & $\widetilde{c}_{21}$ & $\widetilde{c}_{22}$ & $\widetilde{c}_{23}$ & $\tilde{c}_{2 j}$ & $\widetilde{c}_{2 n}$ \\
$\vdots$ & $\vdots$ & $\vdots$ & $\vdots$ & $\cdots$ & $\vdots$ \\
$i$ & $\widetilde{c}_{i 1}$ & $\widetilde{c}_{i 2}$ & $\tilde{c}_{i 3}$ & $\widetilde{c}_{i j}$ & $\widetilde{c}_{i n}$ \\
$\vdots$ & $\vdots$ & $\vdots$ & $\vdots$ & $\cdots$ & $\vdots$ \\
$N$ & $\tilde{c}_{n 1}$ & $\tilde{c}_{n 2}$ & $\tilde{c}_{n 3}$ & $\widetilde{c}_{n j}$ & $\tilde{c}_{n n}$
\end{tabular}

Where $\widetilde{c}_{i j}$ is a non-negative triangular fuzzy number.

For the fuzzy assignment problem, a triangular shaped membership function for fuzzy cost coefficient is denoted by $\mu_{\widetilde{C}_{i j}}\left(c_{i j}\right)$ and is defined as:

$$
\mu_{\widetilde{C}_{i j}}\left(c_{i j}\right)=\left\{\begin{array}{rc}
0, & c_{i j}<l C_{i j} \\
\mu_{\widetilde{C}_{i j}} l\left(c_{i j}\right)=\frac{c_{i j}-l C_{i j}}{m C_{i j}-l C_{i j}}, & l C_{i j} \leq c_{i j} \leq m C_{i j} \\
\mu_{\widetilde{C}_{i j}} r\left(c_{i j}\right)=\frac{r C_{i j}-c_{i j}}{r C_{i j}-m C_{i j}}, & m C_{i j} \leq c_{i j} \leq r C_{i j} \\
0, & c_{i j}>r C_{i j}
\end{array}\right.
$$

Where $\mu_{\widetilde{C}_{i j}} l\left(c_{i j}\right)$ and $\mu_{\widetilde{c}_{i j}} r\left(c_{i j}\right)$ represent the left and right hand side of the triangular membership function, respectively.

For fuzzy assignment problem (S2), formulate the following multi objective linear programming problem with fuzzy cost coefficients as:

$$
\text { S3: } \operatorname{Min}\left\{z_{1}(x), z_{2}(x), \ldots, z_{k}(x)\right\}
$$

Subject to

$$
\begin{gathered}
\sum_{i=1}^{\mathrm{n}} x_{i j}=1, j=1,2, \ldots, n \\
\sum_{j=1}^{\mathrm{n}} x_{i j}=1, i=1,2, \ldots, n \\
x_{i j} \in\{0,1\} \text { for } i, j=1,2, \ldots, n
\end{gathered}
$$


By considering the weighting factor, the multi objective linear programming problem is defined as:

$$
\begin{gathered}
\text { S4: Min }\left\{w_{1} z_{1}(x)+w_{2} z_{2}(x)+\ldots+w_{k} z_{k}(x)\right\} \\
\text { i.e. } \sum_{m=1}^{\mathrm{k}} w_{m} z_{m}(x)
\end{gathered}
$$

Subject to

$$
\begin{gathered}
\sum_{i=1}^{\mathrm{n}} x_{i j}=1, j=1,2, \ldots, n \\
\sum_{j=1}^{\mathrm{n}} x_{i j}=1, i=1,2, \ldots, n \\
x_{i j} \in\{0,1\} \text { for } i, j=1,2, \ldots, n
\end{gathered}
$$

\subsection{Algorithm}

The algorithm for the solution procedure of the proposed approach can be summarized in the following steps:

Step 1: Develop the fuzzy assignment problem as described in (S2).

Step 2: Write the elements of the cost matrix of the assignment problem in the form of triangular fuzzy numbers.

Step 3: Define the triangular shaped membership function of each fuzzy cost coefficient as mentioned in Eq. (3).

Step 4: Formulate the multi objective linear programming problem with fuzzy cost coefficients for fuzzy assignment problem (S3).

Step 5: For different weights develop problem (S4) to get an optimal solution.

Step 6: Find the minimum total fuzzy cost by putting the values of $x_{i j}$ in $\sum_{i=1}^{n} \sum_{j=1}^{n} \widetilde{c}_{i j} x_{i j}$.

\section{Numerical Example}

To illustrate the approach let us consider the following $3 \times 3$ fuzzy assignment problem. The costs $\widetilde{c}_{i j}$ (in dollars) are represented by triangular fuzzy numbers and are shown in following Table 1 :

where

$$
\begin{aligned}
& \widetilde{c}_{11}=(4.5,5,5.5), \quad \widetilde{c}_{12}=(8.1,9,9.9), \quad \widetilde{c}_{13}=(2.7,3,3.3), \quad \widetilde{c}_{21}=(7.2,8,8.8), \quad \widetilde{c}_{22}=(6.3,7,7.7), \quad \widetilde{c}_{23}=(7.2,8,8.8) \\
& \widetilde{c}_{31}=(5.4,6,6.6), \quad \widetilde{c}_{32}=(9,10,11), \quad \widetilde{c}_{33}=(10.8,12,13.2)
\end{aligned}
$$

Find the assignment of persons to jobs that will minimize the total fuzzy cost.

Solution: The fuzzy optimal solution of fuzzy assignment problem by using the proposed approach can be obtained as follows:

Step 1: The given fuzzy assignment problem may be formulated in to the following fuzzy linear programming problem:

$$
\begin{aligned}
\operatorname{Min} Z= & \left((4.5,5,5.5) \mathrm{x}_{11}+(8.1,9,9.9) \mathrm{x}_{12}+(2.7,3,3.3) \mathrm{x}_{13}+(7.2,8,8.8) \mathrm{x}_{21}+(6.3,7,7.7) \mathrm{x}_{22}+(7.2,8,8.8) \mathrm{x}_{23}\right. \\
& \left.+(5.4,6,6.6) \mathrm{x}_{31}+(9,10,11) \mathrm{x}_{32}+(10.8,12,13.2) \mathrm{X}_{33}\right)
\end{aligned}
$$

Subject to

$$
\begin{aligned}
& x_{11}+x_{12}+x_{13}=1 \\
& x_{21}+x_{22}+x_{23}=1 \\
& x_{31}+x_{32}+x_{33}=1 \\
& x_{11}+x_{21}+x_{31}=1 \\
& x_{12}+x_{22}+x_{32}=1 \\
& x_{13}+x_{23}+x_{33}=1 \\
& x_{i j} \in\{0,1\}, i=1,2,3, j=1,2,3 .
\end{aligned}
$$

Step 2: Using step 3 to 5 of proposed approach, we transform the fuzzy assignment problem into the following multi-objective linear programming problem: 


$$
\begin{aligned}
\operatorname{Minimize} & \left(\mathrm{w}_{1}\left(4.5 \mathrm{x}_{11}+8.1 \mathrm{x}_{12}+2.7 \mathrm{x}_{13}+7.2 \mathrm{x}_{21}+6.3 \mathrm{x}_{22}+7.2 \mathrm{x}_{23}+5.4 \mathrm{x}_{31}+9 \mathrm{x}_{32}+10.8 \mathrm{x}_{33}\right)+\right. \\
& \mathrm{w}_{2}\left(5 \mathrm{x}_{11}+9 \mathrm{x}_{12}+3 \mathrm{x}_{13}+8 \mathrm{x}_{21}+7 \mathrm{x}_{22}+8 \mathrm{x}_{23}+6 \mathrm{x}_{31}+10 \mathrm{x}_{32}+12 \mathrm{x}_{33}\right)+ \\
& \left.\mathrm{w}_{3}\left(5.5 \mathrm{x}_{11}+9.9 \mathrm{x}_{12}+3.3 \mathrm{x}_{13}+8.8 \mathrm{x}_{21}+7.7 \mathrm{x}_{22}+8.8 \mathrm{x}_{23}+6.6 \mathrm{x}_{31}+11 \mathrm{x}_{32}+13.2 \mathrm{x}_{33}\right)\right)
\end{aligned}
$$

Subject to

$$
\begin{aligned}
& \mathrm{x}_{11}+\mathrm{x}_{12}+\mathrm{x}_{13}=1, \\
& \mathrm{x}_{21}+\mathrm{x}_{22}+\mathrm{x}_{23}=1 \\
& x_{31}+x_{32}+x_{33}=1, \\
& x_{11}+x_{21}+x_{31}=1, \\
& x_{12}+x_{22}+x_{32}=1, \\
& x_{13}+x_{23}+x_{33}=1, \\
& x_{i j} \in\{0,1\}, i=1,2,3, j=1,2,3 .
\end{aligned}
$$

Solve the above problem for different weights. For example, $w_{1}=0, w_{2}=1, w_{3}=1$

Now above problem reduces to

$$
\text { Minimize }\left(10.5 x_{11}+18.9 x_{12}+6.3 x_{13}+16.8 x_{21}+14.7 x_{22}+16.8 x_{23}+12.6 x_{31}+21 x_{32}+25.2 x_{33}\right)
$$

Subject to

$$
\begin{aligned}
& x_{11}+x_{12}+x_{13}=1, \\
& x_{21}+x_{22}+x_{23}=1, \\
& x_{31}+x_{32}+x_{33}=1, \\
& x_{11}+x_{21}+x_{31}=1, \\
& x_{12}+x_{22}+x_{32}=1, \\
& x_{13}+x_{23}+x_{33}=1, \\
& x_{i j} \in\{0,1\}, i=1,2,3, j=1,2,3 .
\end{aligned}
$$

The above problem is solved by using the TORA package. The solution is presented as follows:

$$
\begin{aligned}
& x_{13}=1, x_{22}=1, x_{31}=1, \\
& x_{11}=x_{12}=x_{21}=x_{23}=x_{32}=x_{33}=0,
\end{aligned}
$$

Step 3: The fuzzy optimal total cost is calculated as:

$$
\begin{aligned}
\widetilde{c}_{13}+\widetilde{c}_{22}+\widetilde{c}_{31}= & (2.7,3,3.3)+(6.3,7,7.7)+(5.4,6,6.6) \\
& =(14.4,16,17.6)
\end{aligned}
$$

In other words the optimal assignment is

$$
1 \rightarrow \mathrm{C}, 2 \longrightarrow \mathrm{B}, 3 \longrightarrow \mathrm{A}
$$

\section{Analysis of the Results and Discussions}

The obtained result can be explained as follows:

1) The total cost is greater than 14.4 and less than 17.6 dollars.

2) Let $c_{T}$ represents the total cost, then the percentage of the favourness for $c_{T}$ is given by $\mu_{T}\left(c_{T}\right) \times 100$, where

$$
\mu_{T}\left(c_{T}\right)=\left\{\begin{array}{rc}
0, & c_{\mathrm{T}}<14.4 \\
\mu_{\mathrm{T}} l\left(c_{T}\right)=\frac{c_{T}-14.4}{1.6}, & 14.4 \leq c_{T} \leq 16 \\
\mu_{T} r\left(c_{T}\right)=\frac{17.6-c_{T}}{1.6}, & 16 \leq c_{T} \leq 17.6 \\
0, & c_{T}>17.6
\end{array}\right.
$$

Table 2 lists the solution for above multi-objective linear programming problem for various weights and it also shows that the solutions are independent of weights $\left(w_{m}, m=1,2,3\right)$. 
Table 3 shows the total assignment cost of the most possible, optimism and pessimism value. So the final result of fuzzy assignment problem is shown in Table 4.

From Table 4 it can be seen that total assignment cost of conventional assignment problem is just the most possible cost of fuzzy assignment problem. Then a conclusion may be that the solution of assignment problem is only a special case of fuzzy assignment problem, and that the paper considers the assignment cost as a fuzzy number is more significant and actual. So the solution obtained by this approach is optimum. This shows the efficiency of our approach.

\section{Conclusions}

In the proposed approach an assignment problem with fuzzy cost coefficients has been solved in order to defeat uncertain environment in the real world situation and relevance to solve it. By the proposed approach fuzzy assignment problem has been transformed into multi-objective linear programming problem where the cost coefficients are fuzzy numbers and also proved that the solutions are independent of weights. To illustrate the proposed approach a numerical example is solved and obtained results are discussed. We have found that in our computational experience the results are as per expectations and satisfying. The proposed approach has the following features:

1) This approach is easy to understand and to apply for finding the optimal solution of fuzzy assignment problem occurring in real life situation.

2) It is easy and simple to use for the decision maker and can be easily implemented to solve other type of problems like, transportation problems, project schedules and network flow problems.

3) This approach solves all types of assignment problems, the minimum assignment problem and the maximum assignment problem.

4) This approach proposes an effective and efficient way for handling the fuzzy assignment problem.

5) This approach solves the fuzzy assignment problem without using any ranking function.

\section{References}

Belmann, R., \& Zadeh, L. A. (1970). Decision making in a fuzzy environment. Management Science, 17, 141-164.

Chen, M. S. (1985). On a fuzzy assignment problem. Tamkang J, 22, 407-411.

Liebman, J., Lasdon, L., Schrage, L., \& Waren, A. (1986). Modeling and Optimization with GINO. The Scientific Press, Palo Alto, CA.

Lin, C. J., \& Wen, U. P. (2004). A labeling algorithm for the fuzzy assignment problem. Fuzzy Sets and Systems, 142, 373-391. http://dx.doi.org/10.1016/S0165-0114(03)00017-4

Liu, L., \& Gao, X. (2009). Fuzzy weighted equilibrium multi-job assignment problem and genetic algorithm. Applied Mathematical Modelling, 33, 3926-3935. http://dx.doi.org/10.1016/j.apm.2009.01.014

Majumdar, J., \& Bhunia, A. K. (2007). Elitist genetic algorithm for assignment problem with imprecise goal. European Journal of operational Research, 177, 684-692. http://dx.doi.org/10.1016/j.ejor.2005.11.034

Sakawa, M., Nishizaki, I., \& Uemura, Y. (2001). Interactive fuzzy programming for two- level linear and linear fractional production and assignment problems: a case study. European Journal of operational Research, 135, 142-157. http://dx.doi.org/10.1016/S0377-2217(00)00309-X

Schrage, L. (1984). Linear, integer and quadratic programming with LINDO. The Scientific Press. Palo Alto, CA.

Taha, H. A. (1992). Operations Research, An Introduction, 5th ed. (Macmillan, New York).

Wang, X. (1987). Fuzzy optimal assignment problem. Fuzzy math, 3, 101-108.

Yaakob, S. B., \& Watada, J. (2009). Fuzzy approach for assignment problem. IEEE, 20-24.

Yang, L., \& Liu, B. (2005). A multi-objective fuzzy assignment problem: New model and algorithm. IEEE International conference on Fuzzy Systems, 551-556.

Ye, X., \& Xu, J. (2008). A fuzzy vehicle routing assignment model with connection network based on priority-based genetic algorithm. World Journal of Modeling and Simulation, 4, 257-268.

Zimmermann, H. J. (1991). Fuzzy Set Theory and Its Applications, $2^{\text {nd }}$ ed., Kluwer Academic Publishers, Boston/Dorgrecht/London. 
Table 1. Fuzzy costs (In Dollars)

\begin{tabular}{|c|c|c|c|c|}
\hline & \multicolumn{3}{|c|}{ Jobs } \\
\hline & & A & B & $\mathrm{C}$ \\
\hline \multirow[t]{3}{*}{ Persons } & 1 & $\widetilde{c}_{11}$ & $\widetilde{c}_{12}$ & $\widetilde{c}_{13}$ \\
\hline & 2 & $\tilde{c}_{21}$ & $\tilde{c}_{22}$ & $\tilde{c}_{23}$ \\
\hline & 3 & $\widetilde{c}_{31}$ & $\tilde{c}_{32}$ & $\tilde{c}_{33}$ \\
\hline
\end{tabular}

Table 2. The solution for above multi-objective linear programming problem for various weights

\begin{tabular}{ccccc}
\hline Sr no. & $w_{1}$ & $w_{2}$ & $w_{3}$ & $\left(x_{11}, x_{12}, x_{13}, x_{21}, x_{22}, x_{23}, x_{31}, x_{32}, x_{33}\right)$ \\
\hline 1 & 0 & 1 & 1 & $(0,0,1,0,1,0,1,0,0)$ \\
\hline 2 & 0 & 1 & 0 & $(0,0,1,0,1,0,1,0,0)$ \\
\hline 3 & 1 & 1 & 0 & $(0,0,1,0,1,0,1,0,0)$ \\
\hline 4 & .2 & .4 & .5 & $(0,0,1,0,1,0,1,0,0)$ \\
\hline 5 & 0 & 1 & .5 & $(0,0,1,0,1,0,1,0,0)$ \\
\hline 6 & 1 & 1 & 0 & $(0,0,1,0,1,0,1,0,0)$ \\
\hline 7 & .2 & 0 & 1 & $(0,0,1,0,1,0,1,0,1,0,0)$ \\
\hline 8 & .5 & .5 & .5 & $(0,0,1,0,1,0,1,0,0)$ \\
\hline 9 & 0 & 0 & .5 & $(0,0,1,0,1,0,1,0,0)$ \\
\hline 10 & .1 & .3 & 1 & $(0,0,1,0,1,0,1,0,0)$ \\
\hline 11 & 0 & .4 & 0 &
\end{tabular}

Table 3. The final result of fuzzy assignment problem

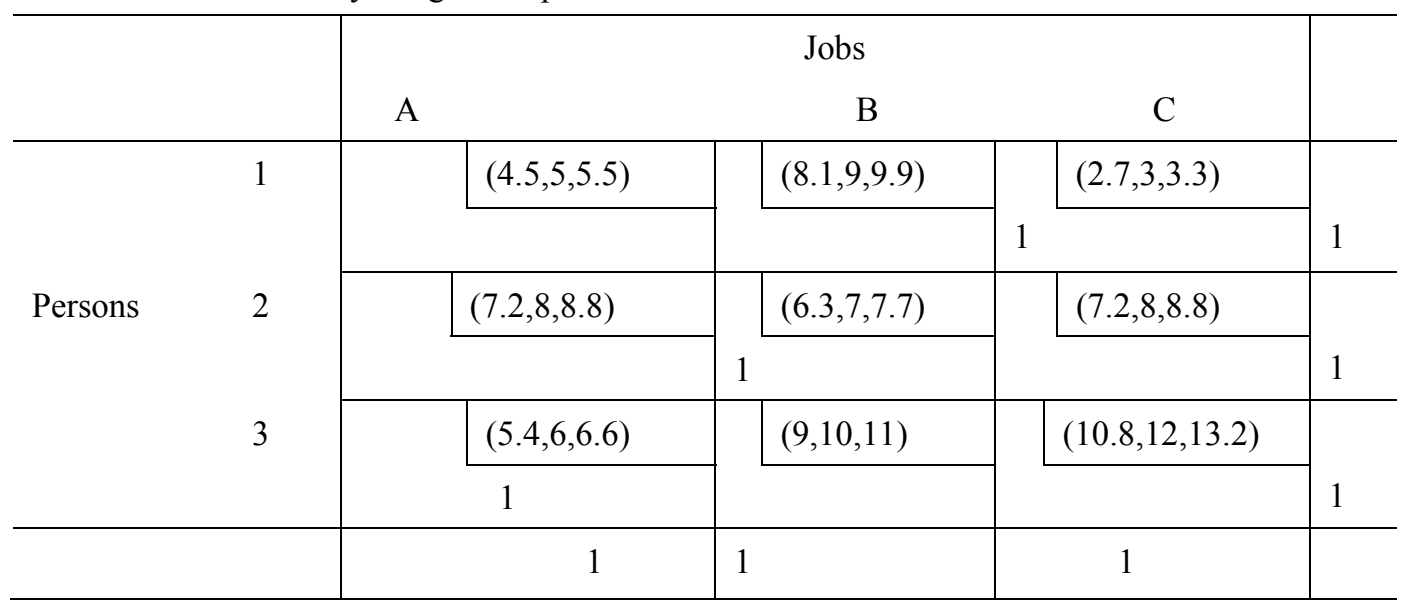


Table 4. Result of conventional assignment problem

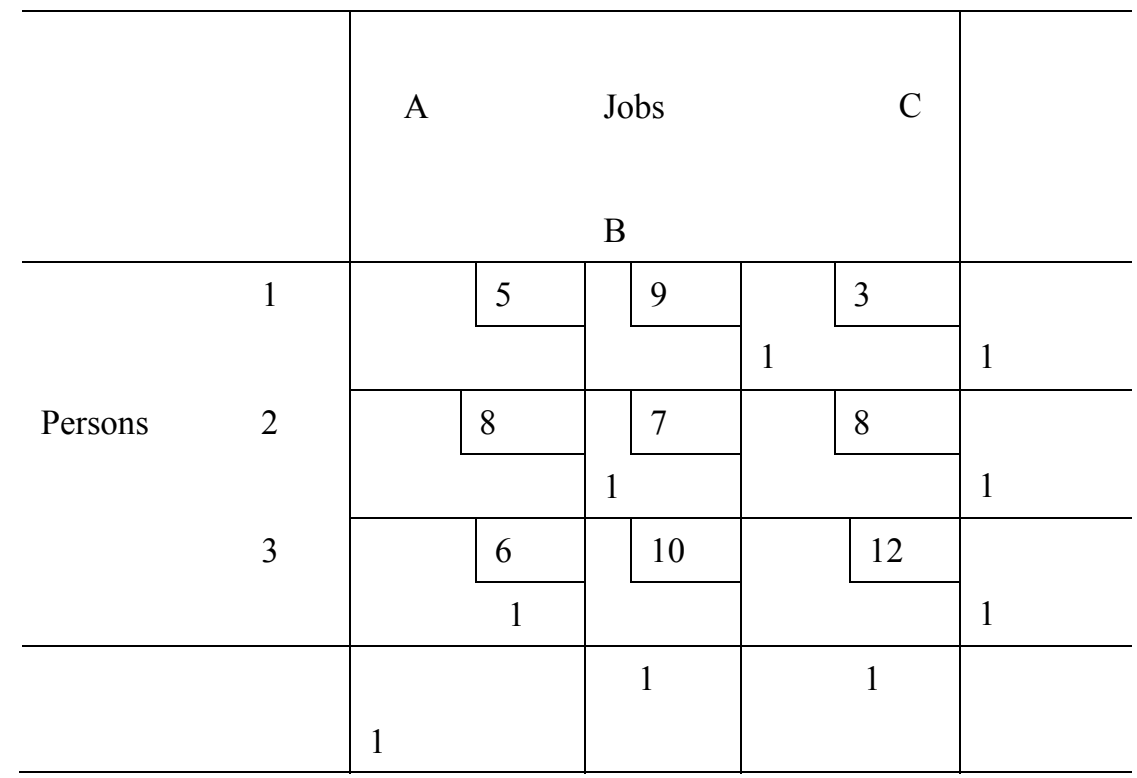

